Rev. Psicol. (Arequipa. Univ. Catól. San Pablo) / Año 2020 / Vol 10 / № 2 / pp. 95-121 ISSN 2306-0565 versión impresa / ISSN 2311-7397 versión on line

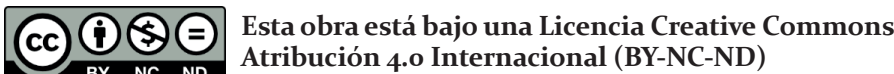

\title{
UNA AGENDA TEMÁTICA PARA LA INVESTIGACIÓN HISTÓRICA DE LA PSICOLOGÍA PARAGUAYA
}

\section{A THEMATIC AGENDA FOR THE HISTORICAL RESEARCH OF PARAGUAYAN PSYCHOLOGY}

\author{
José Emilio García \\ Universidad Católica, Asunción, Paraguay
}

\begin{abstract}
Resumen
En las últimas décadas, la historia de la psicología se consolidó como un campo de investigación cada vez más cultivado por los académicos en el ámbito de la psicología latinoamericana. Los aportes de académicos del continente se refieren tanto a los aspectos relacionados con la disciplina conceptuada al nivel global, como a estudios regionales y los correspondientes desarrollos nacionales. Esto posibilita una indagación sistemática de los procesos y las alternativas seguidas por la psicología en los distintos países de la región. En nuestros días existen estudios intensivos sobre la historia de la psicología en la mayoría de los países latinoamericanos, aunque con un grado de profundización disímil. En Paraguay, las investigaciones históricas referidas a las alternancias, los procesos, eventos, protagonistas y publicaciones centrales que conllevan implicancias históricas se encuentra en aumento, y en la actualidad es posible describir los primeros indicios de una sistematización consistente. Este artículo sumaría la investigación en la historia de la psicología, sus principales ámbitos de indagación y las publicaciones comprendidas en el área, así como las tendencias que ha tomado la investigación y los posibles temas a profundizar en el futuro. $\mathrm{La}$ exposición culmina con una breve revisión de los problemas centrales y los desafíos pendientes. La metodología consiste en un examen crítico de las fuentes publicadas que resultan pertinentes al tema estudiado.
\end{abstract}

Palabras clave: Historia de la Psicología, Paraguay, historiografía, investigación histórica, América Latina. 


\begin{abstract}
In recent decades, the history of psychology strengthened as a field of research due to the increasing involvement of scholars in the area of Latin American psychology. The contributions of academics on the continent both refer to issues related to the discipline conceived at the global level, as well as regional studies and relevant corresponding national developments. This enables a systematic research of the processes and options followed by Psychology in the different countries of the region. Currently, there are intensive studies concerning the history of psychology in the majority of Latin American countries, although these researches are of quite dissimilar depths. In Paraguay, historical research related to the issues, processes, events, actors and the main publications that have some historical implications is increasing, and today it's possible to describe the first indications of a consistent systematization. This paper summarizes research in the history of psychology, its main areas of inquiry and related publications, as well as the trends that impacted research, and possible themes for future exploration. The article concludes with a brief review of the key issues and the challenges ahead. The methodology consists of a critical review of published sources that are relevant to the topic studied.
\end{abstract}

Keywords: History of Psychology, Paraguay, historiography, historical research, Latin America.

\section{El marco de fondo para la psicología y su historia}

Aludir a la indagación histórica de la psicología paraguaya es adentrarse en un ámbito cuya inserción en la disciplina es bastante reciente, en términos de productos publicados. Pero también es apuntar hacia un dominio del conocimiento cuyos equivalentes inmediatos en otras áreas colindantes, que asimismo investigan los orígenes y desarrollo de ciencias vecinas, como la historia de la sociología, la pedagogía o la medicina, y dentro de esta última la psiquiatría, registran un avance incluso más esporádico. En general, el trabajo de los historiadores profesionales en el Paraguay se halla más inclinado, o incluso sesgado, hacia la historia política, bélica y cultural, con algunas presentaciones menores que se enmarcan en el campo de la historia social. Pero se observa un reducido o casi nulo empeño de reconstrucción documental en temas relacionados con la investigación científica y técnica. Como categoría genérica, la historia de la ciencia es una de las ramas que ha logrado menor convocatoria en la preferencia de los historiadores. De hecho, los escritos que se conocen sobre los recorridos temporales de otras especialidades, vecinas o no de la psicología, o de las publicaciones concebidas al interior de esos campos, con el agregado circunstancial de algún ordenamiento cronológico muy elemental, obedecen a los esfuerzos de académicos que no son historiadores profesionales y, por lo 
tanto, no han recibido necesariamente una formación específica en la metodología que utiliza la historiografía científica (Caballero Merlo, 2011; Heisecke, 1965; Meliá \& Palau, 1975). En el pasado inmediato, cabe mencionar el antecedente del historiador Justo Pastor Benítez (1895-1963), quien dio a conocer algunos libros, ahora clásicos, que se enmarcaban en el contexto más amplio que supone la cultura nacional. En ellos, su autor hacía mención a determinados exponentes de la antropología y la sociología, evocando a la vez sus perfiles intelectuales e ideas (Benítez, 1959, 1967).

El supuesto de que únicamente los historiadores profesionales deben investigar el desarrollo temporal de las disciplinas científicas es, de hecho, una afirmación muy discutible. No obstante, también cabe presumir algunas falencias y limitaciones como resultado directo de esta condición, es decir, la ausencia de un entrenamiento técnico especial en los métodos y técnicas de uso corriente en la investigación documental. De esta eventualidad surge el riesgo de que las historias escritas a partir de una perspectiva más interna, es decir, cultivada por aquéllos que representan a la psicología con un sentido de mayor pertenencia profesional, y que se identifican con la misma, pueda conducir a reconstrucciones de tipo celebratorio, en las cuales se asume, por lo general de manera implícita, la existencia de un progreso gradual, que comienza en un pasado remoto y conduce hacia un presente que se estima cualitativamente mejor (Danziger, 1990). La raleada literatura que corresponde a la historia de la ciencia en el Paraguay no es un patrimonio exclusivo de la psicología, o aún de las ciencias comportamentales, sino de todos los sectores del conocimiento. Este es uno de los puntos de partida necesarios para visualizar correctamente la actual situación que nos concierne en este artículo.

Por otra parte, resulta ilustrativo considerar la actitud que mantienen los psicólogos hacia su campo y respecto a la investigación en particular. Pese a algunos problemas muy puntuales que la aquejan, la psicología es una profesión con aceptable éxito social en el Paraguay, y en ella pueden distinguirse ciertas áreas de aplicación que se hallan en gradual pero sostenida expansión, así como una mejora en la calidad de la atención ofrecida. Sin embargo, el peso e importancia que tiene la actividad científica, como sector de elección vocacional para los estudiantes y practicantes jóvenes y lo infrecuente de su producción, se encuentra en abrumadora desventaja ante los escenarios centrados en el despliegue profesional de pericias prácticas. A su vez, la psicología persiste con mayor fuerza en tanto ocupación de servicio para los individuos con dificultades de adaptación o en contextos de aplicación dirigidos al amplio espectro de los escenarios sociales y comunitarios. La psicología entendida como iniciativa dirigida a la comprobación de teorías o el ensayo de innovaciones diagnósticas o terapéuticas, concita una menor atención. Es un hecho que el psicólogo paraguayo, aunque comúnmente reconoce lo imprescindible de la ciencia básica para la viabilidad y consolidación de su profesión, no la estima como una alternativa de peso 
en el abanico de sus posibles ocupaciones laborales. Tal situación no ocurre sin buenas causas, desde luego, aunque esa discusión no constituye el foco central en este artículo. Aunque sí habrá de interesarnos una de sus varias consecuencias: el surgimiento tardío y aún limitado de la investigación psicológica con un carácter temporal e histórico.

El análisis y sistematización sobre el curso temporal de la psicología conlleva un trabajo que se realiza a varios niveles complementarios. Deellos, cuatro pueden estimarse como sus elementos centrales: a) la identificación de los protagonistas, autores, creadores, intérpretes y difusores de un tipo de conocimiento que corresponde catalogar como psicológico, así como aquéllos que adaptaron propuestas científicas desarrolladas en otros ambientes culturales y nacionales a las condiciones prevalecientes en el país, en cualquiera de sus diferentes etapas históricas. Este primer tipo de cuestionamiento hace relación a lo que actualmente se denomina la recepción (Blowers, Cheung, \& $\mathrm{Ru}, 2009$; Dagfal, 2004; Taiana, 2007), asimilación y eventual adaptación del saber científico básico, es decir, aquél cuyo propósito primario es satisfacer la necesidad humana de conocer la realidad circundante, por lo que no muestra una propensión inmediata hacia finalidades prácticas, al menos de una manera muy evidente; b) la dilucidación de los intentos por transpolar la psicología desde su función primordial de información concerniente a la dinámica de la mente y el comportamiento hacia intervenciones concretas emplazadas en la esfera de la conducta y cuya meta fundamental es generar alguna forma de cambio, habitualmente entendida como el despliegue de una experticia cuya ejecución debe considerarse como privativa del psicólogo. En este plano incumben fundamentalmente los presupuestos implícitos que condicionan la visión colectiva de la profesión y los contextos sociales específicos en que éstos se manifiestan, por lo que pueden variar de una colectividad a otra; c) el reconocimiento de factores modeladores en la psicología, combinando a un tiempo las influencias del medio cultural del que proceden las teorías, así como los entornos particulares (locales o nacionales) en los que se verifica la recepción de las ideas y su posterior incorporación, asimilación, transformación o circulación y d) la identificación de periodizaciones o fases que permitan descubrir ciertas constantes en el desarrollo de la psicología, basados en la acción de procesos homogéneos que se reflejan preferentemente en un espacio susceptible de ser delimitado temporalmente y que sirven para disociarla o diferenciarla de las demás.

Variables como la cultura local, los usos, las costumbres, la historia nacional, el idioma, las tradiciones, la predominancia religiosa, la política, el sistema educacional, la autopercepción colectiva, la práctica común del o los lenguajes nativos y la ubicación geográfica son determinantes de primer orden. También la capacidad de apertura de quienes cultivan el conocimiento psicológico hacia las particularidades atinentes a la identidad social mayoritaria y de los grupos étnicos, en especial los indígenas, autóctonos o comunidades 
originarias, sus ideas, acervos y usanzas, sus concepciones particulares sobre la cognición y el comportamiento, y la decisión de incorporarlos como temas legítimos y pertinentes en el amplio abanico de la investigación científica. Danziger (2006) llamó la atención sobre un aspecto diferente y muy importante que concierne a las historias locales, y es la presencia de una psicología que podría suponerse propia de un lugar o un país, versus lo "universal" de la disciplina, que generan eventuales complementariedades o tensiones. Si bien existen y pueden escribirse muchas historias "locales" referidas a países concretos, éstas raramente serían consideradas como representativas a un nivel global; mientras que la psicología estadounidense, que en sentido estricto no es sino otra historia local más, se visualiza como la que incumbe a la psicología como tal, es decir, en un carácter internacional. Esta suposición, y los textos que reproducen la lógica que la acompaña, lo que hacen es enmascarar una historia local como si fuera universal.

Entre quienes deliberaron sobre la psicología en el Paraguay, sólo hallamos dos ejemplos que revelan un interés precursor hacia los asuntos históricos. Los escritos respectivos, sin embargo, no se encuentran referidos a los actores o los procesos atinentes a la psicología producida en el país, sino a la disciplina concebida en un sentido de mayor universalidad. Uno de tales artículos fue escrito en tiempos anteriores al establecimiento de la psicología como carrera independiente en los centros universitarios locales, lo que marca el inicio del periodo preuniversitario
(García, 2005a). Su autor fue Manuel Riquelme (1885-1961), un educador que discutió los constructos básicos en un manual titulado Lecciones de Psicología (Riquelme, 1948), donde sintetizaba lo esencial de las teorías de su época. Era un texto con características y propósitos didácticos. Riquelme no dedicó la totalidad de la obra a una presentación detallada sobre la historia de la psicología, sino que incluyó un ensayo que figuraba como anexo al primer capítulo regular. El tema de esta primera sección era la conciencia, el objeto clásico de estudio para la psicología (García, 2008a). Un segundo trabajo, de estilo algo menos "histórico" que el anterior, pero respetando una cronología temporal en su exposición, y además centrado en la descripción de una escuela contemporánea como la reflexología y sus variantes y antecedentes, se debe al médico Eliseo Sosa Costantini. Él fue uno de los primeros profesores de la carrera de Psicología en la Universidad Católica de Asunción, fundada en 1963. El libro (Sosa Costantini, 1974) constituye una de las excepciones notables de monografías publicadas en Paraguay sobre el enfoque de Iván Pavlov (1849-1936) (García, 2016a), así como de otros investigadores rusos muy relacionados con él. Las contribuciones de Riquelme y Sosa Constantini, aunque ciertamente relevantes, no dejan de transmitir una cierta impresión incidental sobre su producción, habida cuenta que aparecen como ejemplos aislados de la orientación hacia lo histórico en la trayectoria intelectual de sus autores, careciendo de continuidad en estudios o sistematizaciones ulteriores. Por consiguiente, no se distingue nada similar a un 
proyecto de investigación metódica cuyo objetivo fuera la reconstrucción histórica de la psicología.

La recomposición más completa se inicia en los primeros años de la década del 200o. A partir de allí, una serie de trabajos que ahondaban en diferentes temáticas, comenzaron a formar lo que podría denominarse el esqueleto básico para un cuerpo teórico creciente de problematización enfocada en la historia de la psicología paraguaya. Paralelamente, se sumaron de manera temprana algunos informes de revisión en el estado del arte. Un esfuerzo preliminar de organización de la bibliografía fue difundido a mediados del pasado decenio y exploraba la constitución de una incipiente historiografía de la psicología en el Paraguay (García, $2005 \mathrm{~b}$ ). En un inventario de la literatura psicológica que abarca todas las áreas principales de la psicología local realizado un año después, también se incluyó un apartado específico sobre Historia de la Psicología y Epistemología (García, 2006a). El análisis de las publicaciones posteriores se encuentra en fase de elaboración y comprenderá el periodo entre el 2006 y el 2020. Una evaluación más actual sobre los campos de investigación en la disciplina (García, 2020a) incorpora a la historia de la psicología junto a subespecialidades como la psicología clínica, la salud mental, la psicología educacional, la psicología experimental, la psicología de la personalidad y la formación y el entrenamiento profesional del psicólogo, y finamente, la ingesta de sustancias. La nueva productividad generada en la historia de la psicología paraguaya se refleja en el aumento de las citaciones en obras especializadas.

Estudios clásicos como el de los estadounidenses J. G. Beebe-Center y Ross A. McFarland, publicado en Psychological Bulletin (Beebe-Center \& McFarland, 1941) y el argentino Américo Foradori, en La psicología en América (Foradori, 1954) hicieron referencias, en ocasiones relativamente extensas, sobre la situación de la psicología en el Paraguay en los decenios de 1940 y 1950 . Esos trabajos se nutrieron principalmente de contactos epistolares con los referentes del momento y no de fuentes impresas de acceso público, que por aquél tiempo no se hallaban disponibles. Klappenbach (2014) resumió la información contenida en varias de ellas, cubriendo todos los países del continente. Algunas obras que se consideran fundamentales en el lapso más reciente por su sólida documentación, como la de Ardila (1986) con relación al desarrollo de la psicología en América Latina, hicieron menciones aún más concisas. Ciertos volúmenes de autoría colectiva como los de Jacó-Vilela, Lopes da Rocha \& Mancebo (2003), Mardones Barrera (2016), Salas (2014), Thornton (2014) y Vivas (2005) incluyen capítulos sobre la historia de la psicología en el Paraguay escritos por autores del país, y algunos refieren temáticas que competen a la psicología internacional (Jáuregui Camasca, León Donayre \& Rodríguez Rea, 2015). En los últimos diez años, un buen número de revistas como Psicología para América Latina (2009), Revista Interamericana de Psicología (2012), Psicologia em Pesquisa (2014), Universitas Psychologica (2014), 
Revista de Psicología de Arequipa (2015) y la Revista de Psicología (Arequipa, Universidad Católica San Pablo) (2015) recogieron trabajos concernidos con diferentes aspectos de la historia de la psicología en el Paraguay. Complementando lo previamente realizado, este artículo se traza los siguientes objetivos: 1) Relevar las publicaciones en historia de la psicología en el Paraguay; 2) Describir las áreas y problemas centrales que se ubican en la agenda de investigación histórica para la psicología en este país; 3) Proponer criterios de clasificación pertinentes que abarquen el conjunto de la literatura existente y 4) Analizar los posibles rumbos que se abren para exploraciones futuras. La próxima sección de este artículo ofrece una perspectiva de la investigación emprendida y que configura la base de la producción histórica sobre la psicología en el Paraguay.

\section{Los temas investigados en la historia de la psicología paraguaya}

En función a lo anteriormente expuesto, revisaremos en este apartado los principales tópicos y problemas que centran la atención en referencia a la psicología paraguaya, interpretada desde un prisma histórico. Esta indagación, empero, no ha demostrado la sistematicidad muy estructurada que corresponde a los proyectos de vigencia continuada en el tiempo. Debido a su inexistente financiamiento, la investigación ha tomado cursos a veces imprevistos, promovidos más por las decisiones circunstanciales que se instalan en el interés del investigador o en las prioridades que perfilan las convocatorias de diverso tenor que realizan las revistas de psicología. Sin embargo, partiendo de una agrupación de los diferentes artículos y capítulos, y siguiendo como criterio el reconocimiento de líneas comunes que surgen entre ellos, es posible distinguir áreas de concentración temática, haciendo la salvedad que, dependiendo de la flexibilidad de los contenidos, algunos informes puedan ser incluidos en más de una categoría:

a) Historias generales: Los artículos y capítulos que se encuadran en la historia de la psicología paraguaya considerada como una totalidad, discuten las transformaciones introducidas en el discurso psicológico, apoyados sobre un itinerario temporal que comienza en los primeros lustros del siglo XVII. El ámbito inicial se ubica en la educación, coincidente con la fundación de los establecimientos de enseñanza en la entonces colonia española, y cuyo objetivo básico era la formación del clero para su misión de evangelizar a los habitantes de la región (Massare de Kostianovsky, 1968). De forma habitual, los recuentos iniciados en esta época se prolongan en su marco de tiempo hasta los días actuales o colocan como límite a determinados acaecimientos muy cercanos cronológicamente. El análisis comprende los cambios sufridos por la psicología en la vertiente conceptual y las diversificaciones que marcan su estudioy enseñanza. Otro punto resaltante es el de la evolución de las ideas. Necesariamente, una historia global parte de una división en períodos, fases o etapas, describiendo sus correspondientes regularidades $\mathrm{u}$ homogeneidades. Los detalles precisos 
serán abordados en una exposición por separado. De acuerdo a esta conceptualización, son principalmente dos los escritos que comprende esta categoría: el de García (2009), entendido como un compendio de los principales acontecimientos, autores y períodos en la psicología nacional, y un segundo trabajo, focalizado sobre los eventos y los protagonistas centrales que han tenido un lugar reconocible en esa historia (García, 2014a). Asimismo, García (2018a) argumenta sobre la importancia del estudio en particular de la historia de la psicología en el Paraguay, destacando su valor para una compresión de las singularidades y los rasgos comunes con la psicología latinoamericana.

b) Historias locales: El concepto de las historias delimitadas a espacios geográficos circunscritos es relativamente nuevo. En términos generales, suponen la toma de conciencia respecto a la importancia de los aspectos que conciernen al entorno local, y su influencia sobre la recepción, modelamiento y tráfico del conocimiento. Las historias de esta clase igualmente reflejan las alternancias que se producen dentro del marco cultural y social en que se asimila y desarrolla la psicología. El libro editado por Mardones Barrera (2016) sobre diversos aspectos que atañen a los enfoques locales es una buena muestra de la maduración progresiva de los proyectos académicos encauzados hacia esa modalidad particular. En el contexto de la psicología latinoamericana pueden mencionarse varios artículos que ejemplifican correctamente el estilo representado por estas historias. Hasta ahora, en Paraguay se estudiaron dos ejemplos que califican en esa categoría y fueron elaborados con idéntica finalidad. Por una parte, se cuenta con un informe sobre la psicología en la ciudad de Encarnación, ubicada en el sur del país. Ese trabajo reconstruye el proceso de la formación académica en las universidades e identifica a los primeros exponentes de la profesión, contextualizándolos con una sucinta biografía (García, 2011b). Además, se ha dado a conocer una historia local de la psicología en Asunción, introduciendo una exposición temporal más dilatada, que empieza con los antecedentes de la época colonial y llega hasta el periodo moderno. Como se indica en el capítulo respectivo (García, 2016a), gran parte de la historia de la psicología paraguaya, con pocas excepciones, es una historia de la psicología en Asunción, por lo que una separación muy tajante entre ésta y lo que ha ocurrido en otras ciudades del país, es más difícil de lograr en este caso.

\section{c) División en periodos temporales:} Organizar el estudio de la psicología en fases, etapas, ciclos o periodos dispuestos de manera consecutiva y cronológica, constituye un componente auxiliar para descubrir regularidades y aislar tendencias que resultan privativas o son más corrientes en algunos momentos históricos que en otros. García (2014a) argumenta que una parcelación en etapas contribuye a fijar una secuencia con base en una presentación ordenada y coherente de los hechos, conformando un elemento esencial para la identificación de los procesos subyacentes y que se hallan en acción permanente. Estos mismos eventos pueden exhibir rupturas 
y discontinuidades, las que también se manifiestan en los criterios demarcatorios que vayan a adoptarse para separar los ciclos entre sí. En Paraguay, por ejemplo, esto se ha identificado como uno de los obstáculos para determinar a los auténticos pioneros de la psicología (García, 2007). Los parámetros para distinguir diferentes fases en la evolución histórica de la psicología en América Latina varían bastante de un país a otro, por las obvias disimilitudes entre los entornos culturales y científicos, pero también por la disparidad de opiniones que sostienen los autores.

La división más frecuente contempla dos lapsos diferenciados: a) un periodo preuniversitario y b) un periodo universitario y profesional. Durante el primero, que abarca una mayor extensión temporal, se reconoce la existencia de una psicología en fusión con el conocimiento filosófico y, en una medida algo menor, el teológico. El predominio de la filosofía escolástica y aristotélica, común en las antiguas colonias españolas hasta bien entrado el siglo XVIII, se advierte en buena parte de este periodo, y particularmente en sus inicios. Pero desde la década de 1880 comienza a notarse una concepción de los fenómenos psicológicos que sugiere una mayor autonomía, asimilándolos ya no con una intención de estudiar el alma inmaterial como un rezago de las aproximaciones espiritualistas del pasado sino la mente, la conciencia, las emociones, y otras entidades conceptualmente equivalentes, de acuerdo a la terminología utilizada en cada caso. Es por eso que esta etapa se subdivide nuevamente en: 1) Un sub-periodo filosófico-teológico, desde 1609 aproximadamente, en coincidencia con el inicio de la formación de los primeros aspirantes al clero, por entonces la única clase de educación "superior" disponible en el país, y que estudiaban una psicología implícita en la filosofía y la teología según los moldes vigentes en la época; 2) Un sub-periodo de conceptualización autónoma, comenzando hacia 1888 con la progresiva edición de artículos y algunos libros, en los que ya se percibe la absorción y utilización de los nuevos conceptos psicológicos. De manera convencional se acepta como principio la publicación de la obra del médico paraguayo Diógenes Decoud (1857-1920) sobre el hipnotismo desde un punto de vista psicológico, en la ciudad de Buenos Aires (Decoud, 1888). Esta fase cubre setenta y cinco años, hasta 1963, en que se funda la primera carrera de psicología en la Universidad Católica y se inicia el periodo universitario y profesional, que rige hasta nuestros días, y en el cual la psicología se convierte en una profesión liberal. Una periodización alternativa, aunque centrada en la enseñanza de la psicología, fue propuesta por García (2003a) del siguiente modo: a) la psicología en sus relaciones con la filosofía; b) la psicología en los planes de estudio del nivel medio y c) la psicología y la formación de maestros. En el periodo universitario en cuanto tal, se distinguen:a) una etapa de preeminencia de las universidades tradicionales (19641989), b) las primeras experiencias de expansión académica hacia el interior del país (1989-1996) y finalmente, c) el de las nuevas instituciones universitarias. Este esquema ha sido ligeramente modificado 
en fecha reciente, en un trabajo aún no publicado (García, 202ob). Una estrategia relacionada con el propósito de establecer periodizaciones ha sido la adaptación de modelos diseñados para otros contextos culturales y nacionales, como el de Azuma (1984) para la psicología en el Japón, extendiéndolos alámbito específico de la psicología paraguaya (García, 2004).

\section{d) Historias en áreas de la psicología} básica: En sentido amplio, la investigación básica estudia los fundamentos teoréticos y la comprobación de hipótesis a través de la contrastación empírica, aunque sin la pretensión de extender sus resultados a la esfera de las aplicaciones concretas (Hoffman \& Deffenbacher, 1993). La distinción entre psicología básica y aplicada hunde sus raíces en el escrutinio filosófico que busca descubrir la esencia de las cosas, y que entre los griegos se identificó principalmente con la tradición platónica (Muirhead, 2016). Los propósitos aplicados, por otra parte, se orientan según los principios y reglas usuales en la política aristotélica (Schönpflug, 1992). Esta diferenciación, de antiguas raíces, no ha desaparecido de la psicología, sino que, por el contrario, cobra una vigencia muy actual. En el panorama de la disciplina tal como se ha desarrollado en el Paraguay, descubrimos que ciertos dominios temáticos que se ubican en el espectro de la psicología básica recibieron un tratamiento detallado. Se documentaron los orígenes de la psicología social y sus discusiones iniciales como parte de contextos más amplios, en particular las cercanas vinculaciones con la sociología de raíz francesa despuntar el siglo XX.
Se destacan en especial los aportes de dos abogados: Cecilio Báez (1862-1941) e Ignacio A. Pane (1881-1920) (García, 2003b, 2018b). Asimismo se exploraron los comienzos de la psicología experimental como cátedra universitaria, en varias facetas diferentes: a) en su inserción dentro de la carrera de psicología de la Universidad Católica de Asunción (García, 2010a), b) por su presencia en otras instituciones de educación superior en el país (García, 2010b), c) en los recorridos paralelos que ha seguido la aproximación experimental en las demás naciones de la región (García, 2014b) yd) para comprender las probables circunstancias que explican su escaso desarrollo en el Paraguay (García, 2015a). También la psicología política es tema de algunos estudios, abarcando de manera secundaria sus relaciones con un campo de implicancias sociales muy prácticas como la psicología comunitaria (García, 2013a, 2013b, 2016b). Los antecedentes de la psicología de la ciencia, descubiertos en un breve escrito de R. Ross, se exponen en otro artículo (García, 2016c). Finalmente, se analizan las primeras adaptaciones locales de pruebas psicométricas como el test de Stanford-Binet (García, 2016d), en una suerte de puente entre el conocimiento elemental de la inteligencia y la utilización de escalas de medición.

\section{e) Historias en áreas de la psicología} aplicada: Los estudios sobre psicología aplicada en el Paraguay están referidos a tres campos principales: a) la psicología educacional, b) la psicología clínica y c) la psicología de la salud. Los pedagogos y maestros de aula se hallan entre los primeros que reconocieron la importancia 
de las variables que inciden en el desarrollo mental y emocional de los niños y la relevancia de las interacciones entre enseñanza y procesos cognitivos como determinantes para el sostenimiento de la calidad educativa. En este sentido, García (2006b) analizó, no precisamente los cambios en la psicología educacional como sector disciplinario e institucional en cuanto tal, sino las relaciones más generales que se establecen entre psicología y educación, explorando los sustentos teóricos que la primera ha significado para la segunda, además de la evolución de los conceptos y las ideas de sus seis artífices más característicos: Francisco Tapia (1859-1914), Juan Ramón Dahlquist (1884-1956), Ramón Indalecio Cardozo (1876-1943), Manuel Riquelme, María Felicidad González (1884-1980) y Emilio Uzcátegui (1899-1986). En tiempo más próximo, y en un contexto donde se exploran las mallas curriculares vigentes y la formación de los psicólogos educacionales en general, también se consideraron aspectos históricos (García, 2015b).

El desarrollo cronológico y antecedentes principales de la psicología clínica, así como los precursores centrales y las orientaciones predominantes son otros temas analizados (García, 2011a). Las influencias teóricas que desembocaron en la constitución de la psicología clínica paraguaya variaron desde una supremacía inicial clara y definida de la orientación psicoanalítica, sobre todo en la línea freudiana, en las décadas de 1960 y especialmente en la de 1970, hasta una gama conceptual mejor repartida en el decenio de 1980, cuando emergen la terapia del comportamiento y el enfoque sistémico. Los modelos de corte humanista, ya presentes desde finales de la década de 1970, ganaron un pequeño impulso en el tiempo actual, como resultado del desgaste de las aproximaciones tradicionales. Un estudio de Franco Costa (1975), de sólo dos páginas, informa muchos detalles sobre la situación del psicoanálisis e mediados de los setenta, y las escuelas que ostentaban mayor predominio dentro de éste. Otra sub-especialidad que ha merecido una mirada histórica particular es la psicología de la salud (García, 2015c). La reconstrucción empieza con la fundación del primer Manicomio Nacional a comienzos del siglo XX, y sigue con la introducción y difusión institucional del constructo de salud mental en las instancias correspondientes del Ministerio de Salud Pública y Bienestar Social, así como la aparición del concepto de psicología de la salud en el contexto académico de las carreras de psicología. En un área colateral como es la psiquiatría y el cuidado hospitalario, tiene gran mérito un artículo de Cegla \& Franco (1984) que contiene mucha información pertinente sobre las intervenciones a este nivel. En fecha más reciente, Ayala (2016) ensayó una secuenciación de la desmanicomialización en el Paraguay.

\section{f) Representantes o precursores de la psicología en el periodo preuniversi-} tario: El reconocimiento de los autores que cultivaron un discurso psicológico, o difundieron y/o adaptaron enfoques provenientes de la ciencia extranjera, ocupa un lugar preeminente en la investigación de la secuencia temporal de la psicología en el Paraguay. Ciertamente, no 
todos reciben una atención proporcional, y algunos de ellos, juzgados por la mayor significación que se asigna a sus aportes, concentran la mayor cantidad de publicaciones. También son referenciados en coberturas de tinte más general, aunque en esta sección hemos de considerar únicamente los estudios específicos. A quien se ha dedicado una mayoría de los trabajos es Ramón Indalecio Cardozo, cuyas contribuciones destacan por igual en la psicología y la pedagogía. Existen artículos que exploran su ubicación en cuanto pionero de la psicología (García, 20o8b), el rol que cumplió en la introducción tanto de las ideas freudianas (García, 2003c), como de los conceptos psicológicos de William James (García, 2016e), de la escala métrica de la inteligencia del Stanford-Binet (García, 2016d), de los principios de la escuela activa en lo que concierne a los procesos cognitivos (García, 2011c), de la psicología infantil (García, 2010c), y de la vinculación temprana entre psicología y pedagogía -donde es incluido junto a otros exponentes de esa tradición- (García, 2006b), así como recuentos intensivos de su bibliografía (García, 2015d) y la composición y contenido de los libros de psicología en la Biblioteca Pedagógica que había creado para acercar importantes obras de consulta a profesores y alumnos de centros escolares que carecían de colecciones de textos (García, 2016f).

Los ensayos del abogado e historiador Manuel Domínguez (1869-1935) se discuten en muchas diferentes aristas, pero especialmente su visión del carácter nacional (García, 2012a), así como las influencias recibidas del antropólogo alemán Hermann Schaaffhausen (1816-1893) en su pensamiento sobre los determinantes biológicos, ambientales y geográficos de la idiosincrasia de los paraguayos (García, 2016g). También se ha estudiado la dedicación juvenil de Eusebio Ayala (1875-1942), ex-presidente de la República en dos ocasiones no consecutivas (1921 a 1923y 1932 a 1936), a problemas en la gama de la psicología básica (García, 2005c). Las investigaciones del naturalista suizo Moisés Bertoni (1857-1929) sobre la mente de los guaraníes son cubiertas en un par de artículos (García, 2014c, 2016h) y un capítulo (2017a). Al maestro Manuel Riquelme se lo nombra en reconstrucciones históricas generales, pero en particular se abordan sus escritos sobre la historia de la psicología, caracterizados por el paralelo entre la evolución de la ciencia psicológica y los cambios en la antigua noción del alma (García, 2008a). Los dos principales exponentes de la sociología y la psicología social en los comienzos del siglo XX, Cecilio Báez e Ignacio A. Pane, recibieron análisis conjuntos en un par de capítulos incluidos en obras colectivas (García, 2003b, 2018b). Recientemente, y al cumplirse una centuria de publicación de los Apuntes de Sociología de Pane (1917), se evaluaron sus tesis sobre las relaciones de la sociología con la psicología social, la psicología individual y la psicología de las masas (García, 2017b). Igualmente se ha debatido la actuación de Guillermo Enciso en la propagación de las teorías del psiquiatra austríaco Alfred Adler (1870-1937) y la psicología del individuo en el Paraguay (García, 2017c). 
g) Representantes o precursores de la psicología en el periodo universitario: En comparación con los autores que escribieron en la anterior etapa, los trabajos referidos al periodo universitario y profesional se reducen a unos pocos artículos, por lo que constituye el periodo que aún requiere de una exploración más intensiva. Del psicólogo y sacerdote José de Jesús Aguirre (1922-2002) se habla en numerosas fuentes, aunque la repercusión internacional que logró, que puede valorarse por su inclusión entre los académicos que elaboraron reseñas breves sobre sí mismos en el famoso texto de psicología de Whittaker \& Whittaker (1987), ha sido resaltada por García (2014d). Alonso (2015) entrega valiosos datos y recuerdos personales sobre el padre Luis Ignacio Ramallo y su amplio predicamento en los comienzos de la psicología paraguaya, mientras García (2000) hace un relato biográfico de Carlos Luis Lafuente (1944-200o), estimando las influencias intelectuales que recibió durante su entrenamiento fuera del país y la contribución que le cupo en la docencia superior paraguaya. Los tres fueron profesores en la carrera de psicología de la Universidad Católica, aunque Aguirre también desarrolló, hasta su muerte, una extensa labor docente en la Universidad Nacional de Asunción.

\section{h) Historia de la investigación psico-}

lógica: Además de las circunstancias asociadas a la importación, adopción y difusión de las orientaciones teóricas que ganaron espacio en la psicología nacional, la investigación en su doble aspecto de práctica y producto ha concitado algunos estudios. En fecha muy próxima,
García (2020a) analizó las concepciones que prevalecen sobre la investigación, sus variantes metodológicas, aspectos problemáticos y los posicionamientos de diferentes autores sobre sus requerimientos e implementación. Durante el periodo preuniversitario, la investigación y la teorización psicológica interactuaron de maneras específicas, en escenarios que divergen de los actuales en las instancias académicas, y que son analizadas por García (2005a). Otros artículos, aunque no seapoyen en una reconstrucción histórica como su componente más directo, aportan información complementaria para una discusión completa sobre estos problemas (García, 2003d, 2006a, 2012b, 2015a).

\section{i) La enseñanza, la formación acadé-} mica y los currículums de psicología: La enseñanza de la psicología en el Paraguay se ha insertado a tres niveles: a) el de la educación media, b) el de formación de maestros y c) el universitario. Como preludio a un trabajo de exploración que aplica la metodología de juicio crítico de experto e informes publicados, García (2003a) ofreció una sucinta recapitulación del camino recorrido, estableciendo además en ese mismo estudio, la periodización alternativa que se explica en el punto " $e$ ". El análisis histórico de las cátedras se limitó hasta ahora a la de psicología experimental en la Universidad Católica de Asunción (García, 2010a), con referencias complementarias sobre los orígenes de la didáctica de esta área en el entrenamiento de docentes y en el de psicólogos (García, 2010b, 2015a). En un examen del estado de la psicología en Encarnación, ya mencionado 
con anterioridad, se encuentran detalles sobre el comienzo de la psicología en las instituciones universitarias que posee la ciudad y que se encargan de entrenar psicólogos (García, 2011b). Petters (2010) transcribió documentos que incumben a la fundación del primer departamento de psicología en la Universidad Católica en 1963, y García (202ob) investigó las antecedencias y condiciones en que fue creado el currículum original para el mismo centro educativo. Con respecto a la Universidad Nacional de Asunción, García (2010d), en una investigación cuyo eje principal no es el histórico, analizó críticamente la impartición de diez materias de su malla curricular.

\section{j) Enseñanza de la historia de la psico-} logía paraguaya: En años recientes se ha discutido la posibilidad de instituir la enseñanza universitaria de la historia de la psicología paraguaya, delimitando de esta forma un campo propio y a la vez diversificado con respecto al resto de la psicología en América Latina, aunque profundamente asociado con ella. Un par de artículos se refieren a esta problemática. Por un lado, García (2011d) estableció la fundamentación conceptual y filosófica y enumeró los objetivos generales y específicos que resultan pertinentes para una didáctica particular de la historia de la psicología paraguaya como temática diferenciada. Igualmente, en otro trabajo (García, 2011e) se procede a un recuento de los antecedentes, las experiencias realizadas y los ejes de contenido para la efectiva puesta en práctica de un proyecto de enseñanza para la psicología local. Queda pendiente un mayor esclarecimiento de los programas y tópicos para futuros cursos o seminarios. Fierro (2015) apunta a esos mismos estudios cuando revisa una muestra de artículos teoréticos y empíricos sobre la enseñanza de la historia de la psicología en varias naciones latinoamericanas como Argentina, México y Perú y en otras partes del mundo, lo cual ilustra la vigencia actual de estas iniciativas. Pero es preciso aclarar que los ensayos realizados en el Paraguay e incluidos en este parágrafo se dirigen con exclusividad a la psicología de este país.

\section{k) Participación estudiantil: Los estu-} diantes de psicología tuvieron una intervención decisiva, aunque con frecuencia muy ignorada, en los asuntos relacionados a su formación académica. Las iniciativas cristalizaron principalmente en la organización de congresos y sociedades. Gallegos (2009, 2014) y Salas, Ayala, Jibaba \& Nazaret (2014), entre otros, entregan numerosos y abundantes datos sobre esta "psicología de los menores" en nuestro continente. En Paraguay, la historia de la implicación de los alumnos sólo se indaga en un artículo referido a la celebración de la "Tercera Jornada de Estudiantes de Psicología", cuya sede fue la Universidad Católica de Asunción, en 1983. Asimismo, se observa el contexto social y político de la época, con una presentación de las temáticas principales y las concepciones psicológicas que se notaron en aquél evento (García, 2016i).

\section{1) Revistas que incluyen artículos de} orientación psicológica: Las publicaciones especializadas en contenidos psicológicos no son muy abundantes en Paraguay, 
y las que existieron muestran, en su mayoría, trayectorias efímeras. Además, son de aparición muy reciente. De todas maneras, en los inicios del siglo XX, un grupo de ediciones pertenecientes a instituciones con perfiles culturales dieron espacio a elaboraciones teóricas que cabe adscribir, por su temática, como producciones psicológicas. Entre ellas, la más característica es la Revista del Instituto Paraguayo, difundida entre 1896 y 1907 , y sobre la cual se cuenta con un estudio en particular (García, 2014e). Allí se revisan siete artículos impresos entre 1897 y 1903. Por otra parte, la revista Letras, editada entre 1915 y 1916, es objeto de análisis detenidos para los trabajos incluidos en sus páginas y que se relacionan directa o indirectamente con la psicología, tanto por autores del país como del extranjero (García, 2015e, 2016c). Aún queda pendiente el escrutinio de otras publicaciones fundamentales para la configuración de la psicología en el Paraguay.

\section{m) Perspectivas centradas en el carác-} ter nacional: A finales del siglo XIX y comienzos del XX, numerosos escritores e intelectuales, en diversas naciones latinoamericanas, entregaron reflexiones concebidas con diverso tenor valorativo, y con frecuencia imbuidas de apreciaciones muy pesimistas, sobre las condiciones psicológicas que concernían a sus pueblos. Estos autores apelaron al conocimiento científico disponible en su tiempo, en un intento por desentrañar las relaciones entre las mezclas raciales y el intercambio de la sangre autóctona con la del español a lo largo de la conquista, así como los procesos ambientales y climáticos que influían. Todo ello intentando lograr visos de cientificidad en las conclusiones. Estos trabajos poseían, las más de las veces, inocultables connotaciones racistas, reflejo de preconceptos de la época, y de las que casi siempre se responsabilizaba al elemento indígena. No obstante, pueden mencionarse algunos ejemplos de estimaciones positivas. En el Paraguay, la obra de Manuel Domínguez constituye un prototipo muy interesante de esta última vertiente. Las exposiciones más detalladas de su concepción se sitúan en un conjunto de trabajos que analizan cuestiones de mayor generalidad (García, 2013a), así como estudios más focalizados (García, 2012a, 2016g). Sus ideas, que guardan la singularidad de exhibir un tono optimista y casi eufórico en relación a las cualidades exhibidas por los paraguayos, brindan un perfil marcadamente divergente a otras aproximaciones que han sido frecuentes en la psicología latinoamericana. Otros referentes, que también revisten importancia histórica como artífices de un pensamiento que se vincula con esta dimensión de la psicología, fueron objeto hasta ahora de una menor atención (García, 2003b, 2018b).

\section{n) La psicología de los pobladores} autóctonos: La búsqueda de los elementos que determinan el carácter nacional estuvo focalizada sobre las particularidades de los habitantes del territorio paraguayo, concebidos en dos variantes disyuntivas. Por un lado, como descendientes de los primeros colonizadores ibéricos, y por otra, de los pueblos nativos. Los herederos biológicos de estos grupos étnicos se denominan tradicionalmente 
mestizos, con el objeto de diferenciarlos de los criollos, hijos de españoles nacidos en suelo americano (Mora Mérida, 1973). Pero una vertiente distinta indaga las singularidades psicológicas atribuidas a las poblaciones aborígenes, que arribaron a esta región del continente con varios siglos de antelación al desembarco de los conquistadores. Por más que el país se encuentre poblado por diecisiete etnias (Zanardini \& Biedermann, 2001), son los guaraníes los que atraen el mayor grado de atención. Las observaciones sobre las características psicológicas, "morales" y religiosas que se presumen propias de este pueblo han sido reportadas por exploradores europeos, cuya presencia obedecía a muy diferentes razones, a lo largo de más de una centuria, comenzando en los primeros años del siglo XIX. De todos, es Moisés Bertoni el que ha concitado más estudios (García, 2014c, 2016h). Las opiniones del ingeniero español Félix de Azara (1742-1821), el médico francés Alfred Demersay (1815-1891) y el diplomático estadounidense Charles Ames Washburn (1822-1889), además del propio Bertoni, con las variadas perspectivas ideológicas que adoptaron, muy ancladas en los conocimientos y con frecuencia en los preconceptos vigentes, son incorporadas en una investigación reciente (García, 2017a). Otros estudiosos, como el etnólogo italiano Guido Boggiani (1861-1902), que investigó a comunidades residentes en el Chaco paraguayo, recibieron hasta ahora menciones limitadas (García, 2003b, 2018b). El análisis sobre las representaciones de estos núcleos humanos originarios por parte de observadores occidentales guarda no sólo un interés propiamente histórico, sino también una urgencia muy actual, habida cuenta la acelerada desaparición de muchas culturas indígenas en todo el orbe (Rata, Liu \& Hutchings, 2014).

\section{n) Historia de las sociedades cien-} tíficas y profesionales: Un elemento articulador para la conformación de la psicología como espacio de investigación y práctica es la actividad de las sociedades psicológicas. En Paraguay, estas organizaciones no son numerosas en la actualidad ni lo han sido en el pasado, en cantidad ni membresía. Además, en una amplia generalidad de los casos, su acción resulta muy discontinua. No obstante, las circunstancias que rodean su origen y desarrollo, así como sus conexiones más abarcadoras con la comunidad receptora, son un capítulo pendiente. En efecto, la Sociedad Paraguaya de Psicología, a la que puede considerarse la más representativa, aún requiere una reconstrucción exhaustiva, así como la evocación del Día del Psicólogo, muy asociado con ella, y sobre el que solamente existen referencias muy breves (García, 2012c). Respecto a entidades supranacionales como la Sociedad Interamericana de Psicología (SIP), la información disponible es mayor, como se aprecia en un artículo que sintetiza las vinculaciones de ésta con la psicología y los psicólogos paraguayos (García, 2012d).
o) Eventos centrales en la historia nacional paraguaya y sus implicancias para el desarrollo de la psicología como ciencia: La psicología es fruto de sociedades concretas en las que, durante trances históricos bien delimitados, surgen individuos y grupos con intereses 
en su asimilación, adaptación o producción. Por ello, la interacción dinámica de la psicología, como producto elaborado social y culturalmente, mantiene una estrecha interdependencia con sucesos acaecidos fuera de su ámbito estricto, pero en idénticos marcos colectivos. Sin embargo, se trata de una veta muy poco explotada en el amplio contexto temático de la psicología paraguaya. De los grandes episodios que marcaron decisivamente la historia del país, se dispone de un estudio específico (García, 2012e) que analiza las relaciones entre la Guerra contra la Triple Alianza (1865-1870), cuyos efectos fueron devastadores para la población, con el desarrollo regular de la psicología. Se asume, por causas que no precisan argumentarse demasiado, que el conflicto tuvo consecuencias retardatarias sobre el avance científico. La significación de otros hechos que se han dado en el marco de la historia general del país con los destinos de la psicología es recordada más tangencialmente en trabajos separados. Persiste una amplia gama de acontecimientos todavía inexplorados en sus secuelas específicas para la psicología, y los procesos involucrados deberán ser motivo para una indagación sistemática posterior.

\section{Conclusión}

La primera exposición de conjunto sobre la investigación histórica de la psicología paraguaya fue publicada a mediados de la década pasada (García, 2005b). Al momento de aquélla evaluación, la historiografía psicológica constituía un campo de especialización de muy reciente inicio en este país. Comenzaban a delimitarse, más de forma accidental que planificada, lo que serían sus derroteros futuros. A sólo quince años de distancia, el volumen de producción científica aumentó de manera sustancial, recortandoáreas y temas que, en los hechos, configuran una agenda básica. Autores ignorados o poco frecuentados, obras olvidadas y procesos de asimilación desconocidos comenzaron a problematizarse y ser revalorizados paulatinamente. Un conocimiento más claro y completo sobre las transformaciones de la psicología se perfila en los trabajos publicados. Este fortalecimiento no es un componente aislado y único, pues en el ámbito latinoamericano también puede notarse un creciente interés hacia los aspectos concernientes a la evolución de la psicología como ciencia y práctica, así como una consolidación de su contorno investigativo a nivel internacional. Hay varias manifestaciones que permiten reconocer el crecimiento de este empeño. Entre ellos, podría mencionarse el incremento continuo de las publicaciones, el establecimiento de foros especializados, congresos y sociedades, de redes profesionales muy activas, y la aparición de numerosos investigadores jóvenes en casi todos los países. Aunque se carece de un avance parejo y uniforme en las diferentes naciones, y la información disponible es desigual en calidad y cantidad, la tendencia que se insinúa resulta bastante clara. En esta fase de la marcha global de la disciplina, el reconocimiento de líneas concretas por donde encauzar la investigación futura conforma un criterio muy pertinente para una lectura crítica y auto-correctiva de los contenidos producidos y los supuestos adoptados. En lo que 
respecta a la psicología paraguaya, ese ha sido el propósito esencial de este artículo.

Las orientaciones temáticas y los grados de generalidad abarcados demuestran un interesante equilibrio entre las coberturas de carácter más abarcador y la indagación centrada en aspectos particulares y detallados. Esta apertura en diferentes trayectorias de búsqueda posee algunas consecuencias significativas. No implica únicamente una dirección hacia un potenciamiento cada vez mayor de las historias locales, entendidas como los ámbitos temáticos más restringidos en su delimitación, sino además una profundización en sentidos diversos como las condiciones que determinan el nacimiento de vocaciones en los investigadores, los procesos que conducen a la recepción de conceptos y teorías psicológicas, la asimilación de los mismos al entorno local, la réplica o producción activa de investigaciones, y la vinculación de la psicología como producto social con las circunstancias culturales e históricas que son privativas del medio receptor. La investigación en clave temporal es también una forma de análisis fundamentado de la apropiación del conocimiento y las modalidades creativas que tienen las sociedades y los individuos que las componen para fusionar los elementos provenientes de la psicología universal con los de su cultura. Desde este punto de vista, el estudio de la psicología paraguaya guarda connotaciones, no siempre obvias, que desbordan el marco disciplinario más estrecho que le concierne como documentación histórica. Estos intereses son, fundamentalmente, los que definen y orientan la agenda futura. La ampliación de los asuntos esbozados en este artículo y el esclarecimiento de facetas hasta ahora omitidas, así como el surgimiento de nuevos interrogantes, son importantes tareas pendientes. Demostrando una utilidad que vaya más allá de la sencilla reconstrucción de eventos fundamentales en su desarrollo, la capacidad de los estudios históricos de influenciar a la psicología que se despliega tanto en los recintos universitarios como en los espacios profesionales, será lo que finalmente justifique su relevancia y el apoyo que pueda obtener, evitando convertirse simplemente en un campo marginal de poca importancia. Así es como podrá constatarse el alcance real de la historia para la psicología como ciencia y profesión situada en el plano central del interés público. 


\section{Referencias}

Alonso, M. (2015). Luis Ignacio Ramallo y las bases de la psicología científica en el Paraguay. Psicología Básica y Aplicada, 1(1), 20-23.

Ardila, R. (1986). La Psicología en América Latina: Pasado, presente y futuro. México: Siglo XXI.

Ayala, P. (2016). Recuperación histórica de elementos fundadores de la desmanicomialización en Paraguay. Parte I. Psicología Básica y Aplicada, 2(1), 22-34.

Azuma, H. (1984). Psychology in a non-western country. International Journal of Psychology, 19(1), 45-55.

Beebe-Center, J. G., \& McFarland, R. A. (1941). Psychology in South America. Psychological Bulletin, 38(8), 627-667.

Benítez, J. P. (1959). El solar guaraní. Panorama de la cultura paraguaya en el siglo XX. Asunción - Buenos Aires: Nizza.

Benítez, J. P. (1967). Formación social del pueblo paraguayo. Asunción - Buenos Aires: Nizza.

Blowers, G. H., Cheung, B. T., \& Ru, H. (2009). Emulation vs. indigenization in the reception of western psychology in republican China: An analysis of the content. Journal of the History of the Behavioral Sciences, 45(1), 25-33.

Caballero Merlo, J. N. (2011). Cien años de desarrollo de la sociología en Paraguay en el año de su Bicentenario. Del rezago histórico institucional en el pasado a la debilidad contemporánea. Revista Internacional de Investigación en Ciencias Sociales, 7(2), 119-16o.

Cegla, I., \& Franco, F. J. (1984). Historia de la Psiquiatría en el Paraguay. Anales de la Facultad de Ciencias Médicas de la Universidad Nacional de Asunción, 16(1-2), 251-267.

Dagfal, A. (2004). Para una "estética de la recepción" de las ideas psicológicas. Frenia, $4(2), 7-16$.

Danziger, K. (1990). Constructing the subject. Historical origins of psychological research. New York: Cambridge University Press. 
Danziger, K. (2006). Universalism and indigenization in the history of modern psychology. En A. C. Brock (Ed.), Internationalizing the History of Psychology (pp. 208-225). New York: New York University Press.

Decoud, D. (1888). Estudio del hipnotismo. Bajo el punto de vista psicológico y de la medicina legal. Buenos Aires: Félix Lajouane Editor.

Fierro, C. (2015). Estado actual de la enseñanza universitaria de la historia de la psicología. Investigación bibliográfica sobre nueve países occidentales. XVI Encuentro Argentino de Historia de la Psiquiatría, la Psicología y el Psicoanálisis, 16, 195-210.

Foradori, I. A. (1954). La Psicología en América. Buenos Aires: Instituto Cultural Joaquín V. González.

Franco Costa, J. A. (1975). Psicoterapia en Paraguay. Revista Interamericana de Psicología, 9(1-2), 57-58.

Gallegos, M. (2009). Movimiento y participación estudiantil en la psicología latinoamericana: Consideraciones históricas y futuras perspectivas. Avances en Psicología Latinoamericana, 27(1), 33-6o.

Gallegos, M. (2014). La psicología de los menores en América del Sur: Historia del movimiento estudiantil y escenarios futuros. En G. Salas (Ed.), Historia de la Psicología en América del Sur. Diálogos y perspectivas (pp. 291-318). La Serena: Nueva Mirada Ediciones.

García, J. E. (200o). El lugar de Carlos Luís Lafuente (1944-200o) en la psicología paraguaya. Revista Latinoamericana de Psicología, 32(3), 565-570.

García, J. E. (2003a). Problemas centrales en la formación académica y el entrenamiento profesional del psicólogo en el Paraguay. En J. F. Villegas, P. Marassi L. y J. P. Toro C. (Eds.), Problemas centrales en la formación académica y el entrenamiento profesional del psicólogo en las Américas, Volumen II (pp. 205-279). Santiago: Sociedad Interamericana de Psicología.

García, J. E. (2003b). Origens da psicología social no Paraguai. En A. M. Jacó-Vilela, M. Lopes da Rocha y D. Mancebo (Orgs.), Psicologia Social. Relatos na América Latina (pp. 85-122). Sâo Paulo: Casa do Psicologo.

García, J. E. (2003c). Ramón Indalecio Cardozo y la difusión inicial de las ideas de Sigmund Freud en el Paraguay. Teoría e Investigación en Psicología, 11(2), 273-318. 
García, J. E. (2003d). Desafíos para el afianzamiento de la investigación psicológica en el Paraguay. Estudos e Pesquisas em Psicologia, 3(2), 11-36. También puede verse en: http:www2.uerj.br/ revipsi

García, J. E. (2004). La evolución de la psicología en el Paraguay: Una evaluación desde el modelo de Hiroshi Azuma. Revista Intercontinental de Psicología y Educación, 6(2), 25-36.

García, J. E. (2005a). Psicología, investigación y ciencia en el Paraguay: Características resaltantes en el período preuniversitario. Revista Interamericana de Psicología, 39(2), 305-312.

García, J. E. (2005b). Historia e historiografía de la psicología en el Paraguay. En J. R. Vivas (Ed.), Las Ciencias del Comportamiento en los albores del Siglo XXI (pp. 286-290). Mar del Plata: Editorial Universidad Nacional de Mar del Plata.

García, J. E. (2005c). El joven Eusebio Ayala y la psicología paraguaya. Teoría e Investigación en Psicología, 14, 46-90.

García, J. E. (2006a). Publicaciones paraguayas en el área de la psicología: 1960-2005. Revista Latinoamericana de Psicología, 38(1), 149-167.

García, J. E. (2006b). Relaciones históricas entre la psicología y la educación en Paraguay. Psicologia da Educaçâo, 22, 95-137.

García, J. E. (2007). La psicología en Paraguay y el problema de la determinación de los pioneros. Revista Intercontinental de Psicología y Educación, 9(2), 113-146.

García, J. E. (2008a). Manuel Riquelme y la historia de la psicología. Fundamentos en Humanidades, 9(2), 25-54.

García, J. E. (2008b). Ramón Indalecio Cardozo como pionero de la psicología en el Paraguay. Revista Interamericana de Psicología, 42(1), 171-18o.

García, J. E. (2009). Breve historia de la psicología en Paraguay. Psicología para América Latina, 17, Agosto 2009. Recuperado de: http://www.psicolatina.org

García, J. E. (2010a). La cátedra de Psicología Experimental en la Universidad Católica de Asunción: 1985-1987. Revista Interamericana de Psicología, 44(1), 157-167. 
García, J. E. (2010b). La Psicología Experimental en dos universidades paraguayas. Diversitas. Perspectivas en Psicología, 6(1), 81-96.

García, J. E. (2010c). Ramón Indalecio Cardozo y su contribución a la psicología infantil. Boletín de la Fundación «En Alianza», 17(52), 10-13.

García, J. E. (2010d). La enseñanza de la psicología en la Universidad Nacional de Asunción (Paraguay). Teoría e Investigación en Psicología, 19, 61-179.

García, J. E. (2011a). Historia de la Psicología Clínica en el Paraguay. Fundamentos en Humanidades, 12(1), 111-147.

García, J. E. (2011b). Historia y estado actual de la psicología en la ciudad de Encarnación, Paraguay. Fundamentos en Humanidades, 12(2), 37-88.

García, J. E. (2011c). Ramón Indalecio Cardozo: Entre la Psicología, la Pedagogía y la praxis social. En D. Sarah (Coord.), Paraguay: Ideas, Representaciones e Imaginarios (pp. 17-52). Asunción: Secretaría Nacional de Cultura.

García, J. E. (2011d). Enseñanza de la historia de la psicología paraguaya. Pensamiento Psicológico, 9(17), 103-122.

García, J. E. (2011e). La enseñanza de la historia de la psicología en las universidades paraguayas. Revista de Investigación en Psicología, 14(2), 73-96.

García, J. E. (2012a). El carácter nacional del paraguayo en la visión de Manuel Domínguez. Revista Peruana de Psicología y Trabajo Social, 1(1), 143-162.

García, J. E. (2012b). Situación actual de la investigación psicológica en el Paraguay. Cuadernos de Neuropsicología / Panamerican Journal of Neuropsychology, 6(2), 24-38.

García, J. E. (2012c). 22 de mayo: Día del Psicólogo en Paraguay. Blog da Rede Iberoamericana de Pesquisadores em História da Psicologia (RIPeHP). Publicado el 22 de mayo del 2012. Republicado el 23 de mayo del 2014. Recuperado de: http://ripehp.wordpress.com

García, J. E. (2012d). La Sociedad Interamericana de Psicología (SIP) y sus relaciones con la psicología paraguaya. Revista Interamericana de Psicología, 46(1), 51-66. 
García, J. E. (2012e). La Guerra contra la Triple Alianza y su efecto retardatario para la psicología paraguaya. Procesos Históricos: Revista de Historia y Ciencias Sociales, 21, 26-76.

García, J. E. (2013a). Historia y perspectivas de la psicología política en el Paraguay. Les Cahiers de Psychologie Politique, 22, Enero del 2013. Recuperado de: http:// lodel.irevues.inist.fr/cahierspsychologiepolitique/ index/php?ld2347

García, J. E. (2013b). El proyecto de una psicología política en el Paraguay o el equilibrio entre historia, cultura y comportamiento. Les Cahiers de Psychologie Politique, 23, Julio del 2013. Recuperado de:

http://lodel.irevues.inist.fr/cahierspsychologiepolitique/index/php?ld2530

García, J. E. (2014a). Eventos y protagonistas centrales para la historia de la psicología en el Paraguay. En G. Salas (Ed.), Historias de la Psicología en América del Sur. Diálogos y perspectivas (pp. 142-169). La Serena: Nueva Mirada Ediciones.

García, J. E. (2014b). Beginnings and development of Experimental Psychology in five countries of South America. En A. J. Thornton (Ed.), Advances in Experimental Psychology Research (pp. 23-114). New York: Nova Science Publishers.

García, J. E. (2014c). El pensamiento de Moisés Bertoni sobre el origen y la psicología de los indígenas guaraníes. Psicologia em Pesquisa, 8(1), 53-65. Recuperado de: http://www.uff.br/psicologiaempesquisa/home/1678-2

García, J. E. (2014d). La psicología paraguaya representada en la Psicología de James O. Whittaker y Sandra J. Whittaker. Revista Interamericana de Psicología, 48(3), 265-282.

García, J. E. (2014e). Publicaciones psicológicas en la Revista del Instituto Paraguayo. Universitas Psychologica, 13(5), 1815-1833. Recuperado de: http://revistas.javeriana. edu.co/sitio/psychologica

García, J. E. (2015a). Reticencias y paradojas para la consolidación de la psicología experimental en el Paraguay. Cuadernos de Neuropsicología / Panamerican Journal of Neuropsychology, 9(1), 15-40. Recuperado de: http://www.cnps.cl/ index.php/cnps 
García, J. E. (2015b). La formación en Psicología Educacional en el Paraguay. Psicologia da Educaçâo, 41, 3-19. Recuperado de: http://revistas.pucsp.br/index. $\mathrm{php} / \mathrm{psicoeduca}$

García, J. E. (2015c). La Psicología de la Salud en el Paraguay: Historia y desafíos futuros. Revista de Psicología de Arequipa, 5(1), 56-9o.

García, J. E. (2015d). Bibliografía de un educadory pionero de la psicología paraguaya: Ramón Indalecio Cardozo. Revista de Psicología (Arequipa, Universidad Católica San Pablo), 5(1), 87-118.

García, J. E. (2015e). La revista Letras y su contribución a la psicología en el Paraguay. Revista Peruana de Psicología y Trabajo Social, 4(2), 73-97.

García, J. E. (2016a). Historia de la psicología en Asunción: Características y tendencias de su investigación. En R. Mardones Barrera (Ed.), Historia local de la psicología. Discusiones teóricas, metodológicas y experiencias de investigación (pp. 265-301). Santiago: RIL Editores/Editorial Universidad Santo Tomás.

García, J. E. (2016b). La Psicología Política en el Paraguay: Historia, Cultura y Comportamiento. En I. Magaña, A. Dorna \& I. Torres (Eds.), Contribuciones a la psicología política en América Latina: Contextos y escenarios actuales (pp. 401-439). Santiago: RIL Editores.

García, J. E. (2016c). Una temprana psicología de la ciencia en el Paraguay. Propósitos y Representaciones, 4(2), 359-412.

García, J. E. (2016d). La introducción de la escala de inteligencia de Stanford-Binet en el Paraguay. Interacciones: Revista de Avances en Psicología, 2(1), 65-83.

García, J. E. (2016e). La recepción de William James en la obra de Ramón Indalecio Cardozo. Investigaciones en Psicología, 21(3), 53-62.

García, J. E. (2016f). La Biblioteca Pedagógica y las orientaciones psicológicas de Ramón I. Cardozo. Tesis Psicológica, 11(1), 96-115.

García, J. E. (2016g). Influencias de Hermann Schaaffhausen en Manuel Domínguez. Revista de Historia de la Psicología, 37(2), 22-30.

García, J. E. (2016h). De la paleoantropología a la psicología de los guaraníes en la obra de Moisés Bertoni. Revista Peruana de Historia de la Psicología, 2(1), 7-39. 
García, J. E. (2016i). La Tercera Jornada de Estudiantes de Psicología en Asunción (1983). Nuevos paradigmas: Revista Científica de Psicología, 1(1), 9-20.

García, J. E. (2017a). Los estudios psicológicos y antropológicos sobre los indígenas del Paraguay en el siglo XIX y las primeras décadas del siglo XX. En R. E. Mardones (Ed.), Invención de la psique nativa. Construcción de las características psicológicas atribuidas al sujeto indígena en América Latina (pp. 109-200). Santiago: RIL Editores/Editorial Universidad Santo Tomás.

García, J. E. (2017b). Los “Apuntes de Sociología” de Ignacio A. Pane a una centuria de distancia: Análisis de su relevancia histórica para la psicología paraguaya. Revista Peruana de Historia de la Psicología, 3(1), 33-72.

García, J. E. (2017c). Las ideas de Alfred Adler y su asimilación en el Paraguay por Guillermo Enciso. Persona, 20, 29-53.

García, J. E. (2018a). Pertinencia y utilidad de la historia de la psicología paraguaya. Revista Científica de la UMA (Universidad Metropolitana de Asunción), 2, 48-61.

García, J. E. (2018b). Ciento dieciocho años de psicología social en el Paraguay. En A. M. Jacó-Vilela (Ed.), Psicologia Social: Itinerários na América Latina (pp. 147-196). Curitiba: Juruá Editora.

García, J. E. (2020a). La investigación psicológica en el Paraguay: Historia, tradiciones y escenarios del presente. Manuscrito sometido a publicación.

García, J. E. (202ob). El primer currículum universitario de Psicología en la Universidad Católica de Asunción (1963). Manuscrito sometido a publicación.

Heisecke, G. (1965). La bibliografía sociológica en el Paraguay. Revista Paraguaya de Sociología, 1(2), 57-73.

Hoffman, R. R., \& Deffenbacher, K. A. (1993). An analysis of the relations between basic and applied psychology. Ecological Psychology, 5(4), 315-352.

Jacó-Vilela, A. M., Lopes da Rocha, M., \& Mancebo, D. (Orgs.) (2003), Psicologia Social. Relatos na América Latina. Sâo Paulo: Casa do Psicologo.

Jáuregui, D., León, R., \& Rodríguez, M. A. (Eds.) (2015), Homenaje a Reynaldo Alarcón. Lima: Universidad Ricardo Palma, Editorial Universitaria. 
Klappenbach, H. (2014). Informes norteamericanos sobre la Psicología en América del Sur anteriores a 1950. En G. Salas (Ed.), Historias de la Psicología en América del Sur. Diálogos y perspectivas (pp. 228-248). La Serena: Nueva Mirada Ediciones.

Mardones, R. (Ed.) (2016), Historia local de la psicología. Discusiones teóricas, metodológicas y experiencias de investigación. Santiago: RIL Editores/Editorial Universidad Santo Tomás.

Massare de Kostianovsky, O. (1968). La instrucción pública en la época colonial. Asunción: Edición de la autora.

Meliá, B., \& Palau, T. (1975). Producción sociológica sobre el Paraguay. Relevamiento bibliográfico de los últimos años. Estudios Paraguayos, 3(1), 147-173.

Mora Mérida, J. L. (1973). Historia social de Paraguay, 160o-165o. Sevilla: Escuela de Estudios Hispano-americanos de Sevilla.

Muirhead, J. H. (2016). The platonic tradition in Anglo-Saxon philosophy: Studies in the history of idealism in England and America. New York: Routledge.

Pane, I. A. (1917). Apuntes de Sociología. Asunción: España.

Petters, E. (2010). Origen y evolución de la carrera de Psicología. Cuadernos Pedagógicos, Noviembre del 2010, 55-81.

Rata, A., Liu, J. H., \& Hutchings, J. (2014). Creation narratives as metaphors for indigenous identity development: The Pōwhiri identity negotiation framework. Psychology and Developing Societies 26(2), 291-319.

Riquelme, M. (1948). Lecciones de Psicología. Buenos Aires: Ángel Estrada Editores, Novena edición (publicación original: 1936).

Salas, G. (Ed.) (2014). Historias de la Psicología en América del Sur. Diálogos y perspectivas. La Serena: Nueva Mirada Ediciones.

Salas, G., Ayala, J., Jibaba, C., \& Nazaret, F. (2014). Una historia reciente: Los congresos latinoamericanos de estudiantes de psicología (2007-2013). En G. Salas (Ed.), Historia de la Psicología en América del Sur. Diálogos y perspectivas (pp. 321-342). La Serena: Nueva Mirada Ediciones. 
Schönpflug, W. (1992). Applied Psychology: Newcomer with a long tradition. Applied Psychology: An international review, 42(1), 5-30.

Sosa Costantini, E. (1974). Reflexología: La raíz histórica de la reflexología. Asunción: Edición del autor.

Taiana, C. (2007). Transatlantic migration of the disciplines of the mind. Examination of the reception of Wundt's and Freud's theories in Argentina (pp. 34-55). In A. C. Brock (Ed.), Internationalizing the History of Psychology. New York: New York University Press.

Thornton, A. J. (Ed.) (2014), Advances in Experimental Psychology research. New York: Nova Science Publishers.

Whittaker, J. O., \& Whittaker, S. J. (1987). Psicología. México DF: Editorial Interamericana, Cuarta edición.

Vivas, J. R. (Ed.) (2005), Las Ciencias del Comportamiento en los albores del Siglo XXI. Mar del Plata: Editorial Universidad Nacional de Mar del Plata.

Zanardini, J., \& Biedermann, W. (2001). Los indígenas del Paraguay. Asunción: Centro de Estudios Antropológicos de la Universidad Católica.

Recibido: 23 de mayo del 2020

Aceptado: 18 de julio del 2020 